\title{
Topological Rigidity of Strong Stable Foliations for Cartan Actions
}

\author{
Steven Hurder* \\ Department of Mathematics (m/c 249) \\ University of Illinois at Chicago \\ 851 S. Morgan St. \\ CHICAGO, IL 60607-7045 USA \\ email: hurder@boss.math.uic.edu
}

March 9, 2002

\begin{abstract}
We show that the strongest stable foliations associated to the generators of a Cartan action on a compact infra-nilmanifold are invariant under topological conjugacy. This has the corollary that a Cartan action on a compact infra-nilmanifold with constant exponents is smoothly conjugate to an affine action.
\end{abstract}

* Supported in part by NSF Grant DMS 91-03297

${ }^{1}$ AMS classification: Primary 58F15, 58F18; Secondary 57R30 


\section{Main Theorem}

It is a basic property of an Anosov diffeomorphism $\phi: X \rightarrow X$ on a compact manifold $X$ that the stable and unstable foliations, $\mathcal{F}^{s}$ and $\mathcal{F}^{u}$ respectively, are topologically defined in terms of attracting orbits for the forward and backward maps, respectively ([1], or Theorem 6.2, [26]). One consequence is that if $\phi_{0}, \phi_{1}: X \rightarrow X$ are Anosov diffeomorphisms and $H: X \rightarrow X$ is a homeomorphism conjugating $\phi_{1}$ to $\phi_{0}$, then $H$ maps the stable foliation $\mathcal{F}_{1}^{s}$ of $\phi_{1}$ to the stable foliation $\mathcal{F}_{0}^{s}$ of $\phi_{0}$, and similarly for the unstable foliations.

When the stable foliation of an Anosov diffeomorphism $\phi$ has dimension greater than one, the choice of a Riemannian metric or just a Finsler on $T X$ sometimes allows to distinguish a "strongest stable" 1-dimensional subfoliation $\mathcal{F}^{s s}$ of the stable foliation $\mathcal{F}^{s}$. This is a metric property on the differentiable dynamics of $\phi$ (see Definition 2.2 below), and is not a priori a topological invariant of the Anosov diffeomorphism. In the simplest case, a local topological conjugacy between two linear contracting maps need not preserve the rates of contractions, so in particular need not preserve the direction of fastest contraction. In a global setting, a topological conjugacy between Anosov diffeomorphisms $\phi_{1}$ and $\phi_{0}$ of a compact manifold maps each invariant curve for $\phi_{1}$ to an invariant topological curve for $\phi_{0}$. The existence of such curves has been studied by Hirsch [10] and Hancock [9], and Example 7.3 below (due to de la Llavé) shows that non-trivial examples exist.

In this paper we show that with additional hypotheses on the Anosov diffeomorphism - that there is a sufficiently large commuting family of Anosov diffeomorphisms, and $X$ is an infra-nilmanifold - then the strongest stable foliation is topologically invariant. Our proof of this is based on an addendum to the above comments: if $X$ is an infra-nilmanifold, then the rates of expansion of 1-dimensional expanding submanifolds are topological invariants of the action.

A $C^{r}$-action $\varphi: \mathcal{A} \times X \rightarrow X$ of an abelian group $\mathcal{A}$ on a manifold $X$ of dimension $n$ is called Cartan [12] if $\mathcal{A}$ is generated by $\Delta=\left\{\gamma_{1}, \ldots, \gamma_{n}\right\}$ such that each $\varphi\left(\gamma_{i}\right)$ is an Anosov diffeomorphism of $X$ with a 1-dimensional strongest stable foliation $\mathcal{F}_{i}$ and the collection $\mathcal{T}=\left\{\mathcal{F}_{1}, \ldots, \mathcal{F}_{n}\right\}$ forms a trellis for $X$ (cf. section 2.) The generators $\Delta$ need not freely generate $\mathcal{A}$, which can have rank less than $n$.

A manifold $X$ is an infra-nilmanifold if there is a finite cover $\tilde{X}$ which is diffeomorphic to a quotient $\Lambda \backslash \mathcal{N}$ of a simply-connected, nilpotent Lie group $\mathcal{N}$ by a cocompact lattice $\Lambda \subset \mathcal{N}$.

THEOREM 1.1 Let $\mathcal{A}$ be a free abelian group, and $\left(\varphi_{0}, \Delta\right)$ and $\left(\varphi_{1}, \Delta\right)$ be two Cartan $C^{1}$-actions on a compact infra-nilmanifold $X$. Suppose that there exists a homeomorphism $H: X \rightarrow X$ conjugating the action of $\varphi_{1}$ to $\varphi_{0}$. Then for each $1 \leq i \leq n, H$ maps the strongest stable foliation of $\varphi_{1}\left(\gamma_{i}\right)$ to that of $\varphi_{0}\left(\gamma_{i}\right)$.

A trellis $\mathcal{T}$ is maximal (cf. Definition 2.13 [12]) if each foliation $\mathcal{F}_{i}$ is the stable foliation of $\varphi\left(\gamma_{i}\right)$. Theorem 1.1 is immediate in this case. Similarly, when each foliation $\mathcal{F}_{i}$ is the intersection of the stable foliations for a collection of Anosov diffeomorphisms $\{\varphi(\gamma)\}$ with $\gamma \in \mathcal{A}$, it is clear that the trellis structure is preserved by a topological conjugacy. Corollary 3.4 below shows that for a Cartan action on an infra-nilmanifold there is exactly one additional case: each foliation $\mathcal{F}_{i}$ of the trellis is the intersection of center-stable and stable foliations of a collection of diffeomorphisms $\{\varphi(\gamma)\}$ for some $\gamma \in \mathcal{A}$. The center-stable foliation of an arbitrary diffeomorphism is not a priori invariant under a homeomorphism.

Theorem 1.1 has an application to the smooth classification of Cartan actions. Assume that the 1-dimensional tangential distributions $\left\{T \mathcal{F}_{1}, \ldots, T \mathcal{F}_{n}\right\}$ to the foliations of the trellis are orientable, then a choice of unit vector field in each $T \mathcal{F}_{i}$ determines a global framing of $T X$. (If the foliations are not orientable, then we pass to a finite covering of $X$ to obtain this, and formulate our conditions below for the lift of the action to this cover.) Write the derivative of the group transformations $D \varphi(\gamma)$ for $\gamma \in \Gamma$ as a $G L(n, \mathbf{R})$-valued continuous cocycle

$$
D \varphi: \mathcal{A} \times X \longrightarrow G L(n, \mathbf{R})
$$


The Cartan hypotheses on $\varphi$ implies that the image of $D \varphi$ consists of diagonal matrices for all $x \in$ $X$. The diagonal entries of $D \varphi(\gamma, x)$ with respect to the trellis framing yield $\mathbf{R}^{*}$-valued multiplicative cocycles $\left\{\lambda_{1}, \ldots, \lambda_{n}\right\}$. For each periodic point $x \in \operatorname{Per}(\varphi(\mathcal{A}))$, let $\mathcal{A}_{x} \subset \mathcal{A}$ be its isotropy subgroup with corresponding linear isotropy representation

$$
D_{x} \varphi: \mathcal{A}_{x} \rightarrow G L(n, \mathbf{R})
$$

A Cartan action is said to have constant exponents if the representations $D_{x} \varphi$ are constant. That is, there exists a diagonal representation $\rho: \mathcal{A} \rightarrow G L(n, \mathbf{R})$ so that for each $x \in \operatorname{Per}(\varphi(\mathcal{A}))$ and $\gamma \in \mathcal{A}_{x}$, $D_{x} \varphi(\gamma)=\rho(\gamma)$. Theorem 2.21 of [12] showed that a Cartan action on a torus with constant exponents is $C^{r}$-conjugate to an affine action. The same method of proof, with the added information given by Theorem 1.1 and Proposition 3.1, yields a similar result for actions on infra-nilmanifolds:

THEOREM 1.2 Let $\varphi: \mathcal{A} \times X \rightarrow X$ be a Cartan $C^{r}$-action with constant exponents on a compact infra-nilmanifold $X$, for $r=1, \infty$ or $\omega$. Then there is a $C^{r}$-conjugacy of $\varphi$ to an affine action on $X$.

There are many natural examples of Cartan actions. Section 7 of [12] gives an extensive list linear Cartan actions on $\mathbf{T}^{n}$. The thesis of N. Qian [23] describes many examples of Cartan actions on nilmanifolds. An affine translational component can be added to a linear action to obtain an affine Cartan action without fixed-points [13]. Non-affine Cartan actions are obtained from a $C^{1}$-perturbation of a Cartan action, which is again Cartan by Proposition 2.17 of [12].

We conclude this paper by describing three interesting examples. The first, due to Qian, describes a Cartan action on a nilmanifold. Given the additional work required to prove Theorem 1.1 in the nilmanifold case, it is reassuring to have explicit examples. The second example, also suggested by Qian, shows that the theorem does not reduce to the known topological stability of the stable foliations. The last example is due to R. de la Llavé, and exhibits an analytic Anosov map which preserves an analytic trellis and is conjugate to a linear Anosov map, but the conjugacy does not preserve the invariant trellis.

The author is indebted to A. Katok for pointing out the examples of R. de la Llavé, to N. Qian for his explanation of the examples of his Thesis, and to C. Croke for some helpful insight on the geometry of nilmanifolds. The support of the Mathematical Sciences Research Institute in Berkeley during the completion of this paper is gratefully acknowledged.

\section{Cartan actions}

We recall two notions related to the definition of Cartan actions, and a result about their topological classification.

DEFINITION 2.1 ([12]) $A C^{1}$-trellis $\mathcal{T}$ on $X$ is a collection of 1-dimensional, pairwise-transverse foliations $\left\{\mathcal{F}_{i} \mid 1 \leq i \leq n\right\}$ of $X$ such that

1. The tangential distributions have internal direct sum $T \mathcal{F}_{1} \oplus \cdots \oplus T \mathcal{F}_{n} \cong T X$;

2. For each $x \in X$ and $1 \leq i \leq n$, the leaf $L_{i}(x)$ of $\mathcal{F}_{i}$ through $x$ is a $C^{1}$-immersed submanifold of $X$;

3. The $C^{1}$-immersions $L_{i}(x) \hookrightarrow X$ depend uniformly Hölder continuously on the basepoint $x$, for the $C^{1}$-topology on immersions. 
DEFINITION 2.2 An Anosov diffeomorphism $f$ has a one-dimensional strongest stable distribution if there exists a $D f$-invariant, 1-dimensional vector subbundle $E^{s s}$ of the stable distribution which satisfies an exponential dichotomy: that is, there exists

- a Finsler on TX,

- a continuous splitting of the tangent bundle into Df-invariant subbundles, $T X \cong E^{c s} \oplus E^{s s}$,

- constants $0<\mu<\lambda<1$

such that for for all positive integers $m$,

$$
\begin{aligned}
& \left\|D\left(f^{m}\right)(v)\right\|>\lambda^{m} \cdot\|v\| ; \quad 0 \neq v \in E^{c s} \\
& \left\|D\left(f^{m}\right)(v)\right\|<\mu^{m} \cdot\|v\| ; \quad 0 \neq v \in E^{s s} .
\end{aligned}
$$

The strongest stable distribution $E^{s s}$ is necessarily integrable, and the leaves of the resulting foliation $\mathcal{F}^{s s}$ are $C^{1}$-immersed 1-dimensional submanifolds (cf. Theorem IV.1, [26]).

DEFINITION 2.3 ([12]) Let $\mathcal{A}$ be an abelian group with a given set of generators $\Delta=\left\{\gamma_{1}, \ldots, \gamma_{n}\right\}$. Then $(\varphi, \Delta)$ is a Cartan $C^{1}$-action on the n-manifold $X$ if:

- $\varphi: \mathcal{A} \times X \rightarrow X$ a $C^{1}$-action on $X$

- each $\varphi\left(\gamma_{i}\right)$ is Anosov with a 1-dimensional strongest stable foliation $\mathcal{F}_{i}^{s s}$

- the collection of 1-dimensional foliations $\left\{\mathcal{F}_{1}^{s s}, \ldots, \mathcal{F}_{n}^{s s}\right\}$ form a $C^{1}$-trellis on $X$.

It is a straightforward consequence of the work of Franks [6,5] and Newhouse [21] that an Anosov action of an abelian group on a torus with a common fixed-point is topologically equivalent to an algebraic action. This result is also true for Anosov actions on infra-nilmanifolds (Proposition 2.18, [12]) using the corresponding work of Manning [19]:

PROPOSITION 2.4 Let $(\varphi, \Delta)$ be a Cartan $C^{1}$-action on the closed n-dimensional infra-nilmanifold $X$. Then $\varphi$ has a periodic point $x_{0}$, and there is a positive integer $p$ so that the action of the $p^{\text {th }}$-powers $\Delta^{p}=\left\{\gamma_{1}^{p}, \ldots, \gamma_{m}^{p}\right\}$ is topologically conjugate to a standard (algebraic) Cartan action induced by the map on homotopy, $\varphi_{\#}: \Delta^{p} \times \pi_{1}\left(X ; x_{0}\right) \rightarrow \pi_{1}\left(X ; x_{0}\right)$.

There are examples of abelian Anosov actions which do not have a fixed-point [13], so that the reduction to the subgroup generated by $\Delta^{p}$ may be necessary.

\section{Topological invariance of the exponents}

PROPOSITION 3.1 Let $\varphi: \mathcal{A} \times X \rightarrow X$ be a $C^{1}$-Cartan action on a nilmanifold $X$ with a fixed-point $x_{0} \in X$, such that the trellis tangential distributions $\mathbf{E}_{i}=T \mathcal{F}_{i}$ are orientable, and the action of $D \varphi(\gamma)$ is orientation-preserving on $\mathbf{E}_{i}$ for all $\gamma \in \mathcal{A}$ and $1 \leq i \leq n$. Suppose that $H: X \rightarrow X$ is a topological conjugacy between $\varphi$ and a standard affine action $\varphi_{\#}$. Then $\varphi_{\#}$ is Cartan.

We first prove the Proposition in the case where $X \cong \mathbf{T}^{n}$. The non-toroidal case follows by an inductive application of this first step.

Recall the definition of the asymptotic homology class $A_{i} \in H_{1}\left(\mathbf{T}^{n} ; \mathbf{R}\right)$ associated to a typical leaf of $\mathcal{F}_{1, i}$ (due to Schwartzman [25] for the case of 1-dimensional foliations; cf. also Plante [22] and RuelleSullivan [24]). The leaves of $\mathcal{F}_{1, i}$ are $C^{1}$-immersed submanifolds, so the Riemannian metric on $T \mathbf{T}^{n}$ induces a Riemannian metric on each leaf, with corresponding distance function. Chose a sequence of 
connected intervals $J_{m}$ contained in the leaves of $\mathcal{F}_{1, i}$ and let $d_{m}$ denote the length of $J_{m}$. Integration against $J_{m}$ defines a 1-current $\left[J_{m}\right]$ on the 1 -forms $\Omega^{1}\left(\mathbf{T}^{n}\right)$. Rescale each current $\left[J_{m}\right]$ by its length $d_{m}$ to obtain a sequence of norm-one currents $\left\{\frac{1}{d_{m}}\left[J_{m}\right] \mid m>0\right\}$. If $d_{m}$ tends to infinity with $m$, there is a unique weak-* limit of these currents which defines a closed 1-current $\left[J_{*}\right]$ on $\mathbf{T}^{n}$. Denote its homology class by $A_{i}$.

The definition of $A_{i}$ depends upon the choice of a Riemannian metric on $T \mathbf{T}^{n}$, but different choices result in projectively equivalent classes. That is, if $A_{i}^{\prime}$ is the asymptotic class for a second metric, then there exists a non-zero constant $C_{i}$ so that $A_{i}=C_{i} \cdot A_{i}^{\prime}$.

The proposition for the toral case follows by estimating the action $\varphi_{*}(\gamma)$ on the homology classes $\left\{A_{1}, \ldots, A_{n}\right\}$ in terms of the uniform expansion and contraction estimates for $D \varphi(\gamma)$. Let $T \mathbf{T}^{n} \cong$ $\mathbf{E}_{1} \oplus \cdots \oplus \mathbf{E}_{n}$ be the splitting of $T X$ into line subbundles corresponding to the tangent spaces to the trellis foliations. The Cartan assumption on $\varphi$ implies that for each $1 \leq i \leq n$, there exists constants $0<\lambda_{i, k}$ and $0<\mu_{i, k}$ for $1 \leq k \leq n$, a permutation $k \mapsto \pi_{i}(k)$ so that

$$
\lambda_{i, i}<\mu_{i, i}<\lambda_{i, \pi_{i}(2)}<\cdots<\mu_{i, \pi_{i}(n-1)}<\lambda_{i, \pi_{i}(n)}<\mu_{i, \pi_{i}(n)}
$$

and

- the spectrum of $D \varphi\left(\gamma_{i}\right)$ restricted to the subbundle $\mathbf{E}_{k}$ is pinched between $\lambda_{i, k}$ and $\mu_{i, k}$

- $\mu_{i, \pi_{i}\left(k_{i}\right)}<1<\lambda_{i, \pi_{i}\left(k_{i}+1\right)}$ for some $k_{i}$

- $\pi_{i}(1)=i$ for all $1 \leq i \leq n$; that is, $\mathbf{E}_{i}$ is the maximally contracting direction for $\varphi\left(\gamma_{i}\right)$.

Fix a generator $\gamma_{i}$ of $\mathcal{A}$. Choose a sequence of connected intervals $J_{m}$, with each $J_{m}$ contained in some leaf of $\mathcal{F}_{k}$ and having lengths $d_{m}$ tending to infinity. The weak-* limit of the currents $\left\{\frac{1}{d_{m}}\left[J_{m}\right] \mid m>0\right\}$ represents the homology class $A_{k}$, so the homology class of the weak-* limit of the currents $\left\{\frac{1}{d_{m}}\left[\varphi\left(\gamma_{i}\right)\left(J_{m}\right)\right] \mid m>0\right\}$ equals $\varphi\left(\gamma_{i}\right)_{*}\left(A_{k}\right)$. The length of $\varphi\left(\gamma_{i}\right)\left(J_{m}\right)$ is estimated by

$$
\lambda_{i, k} \cdot d_{m} \leq\left|\varphi\left(\gamma_{i}\right)\left(J_{m}\right)\right| \leq \mu_{i, k} \cdot d_{m}
$$

so that $\varphi\left(\gamma_{i}\right)_{*}\left(A_{k}\right)=\lambda_{i, k}^{*} \cdot A_{k}$ where $\lambda_{i, k} \leq \lambda_{i, k}^{*} \leq \mu_{i, k}$.

We deduce that each $A_{k}$ is a non-zero eigenvector of $\varphi\left(\gamma_{i}\right)_{*}$, with $A_{i}$ spanning its 1-dimensional eigenspace of maximal contraction. Thus, the standard induced linear action of $\varphi(\mathcal{A})_{*}$ on

$$
\mathbf{T}^{n} \cong H_{1}\left(\mathbf{T}^{n} ; \mathbf{R}\right) / H_{1}\left(\mathbf{T}^{n} ; \mathbf{Z}\right)
$$

is Cartan. This implies that any affine action whose associated linear representation is the standard action $\varphi(\mathcal{A})_{*}$ is Cartan.

Now consider the case where $X$ is a compact nilmanifold. Theorem 2.2 of Franks ([6]; cf. also [20]) implies that the action $\varphi$ admits a factorization onto a hyperbolic toral action $\varphi_{*}$

$$
\begin{array}{cll}
\varphi: \mathcal{A} \times X_{1} & \longrightarrow & X_{1} \\
\pi \downarrow & & \pi \downarrow \\
\varphi_{*}: \mathcal{A} \times \mathbf{T}^{k_{1}} & \longrightarrow & \mathbf{T}^{k_{1}}
\end{array}
$$

(where we set $X=X_{1}$ to simplify the subsequent notation.)

A key point of Franks' construction is that the map $\pi$ to $\mathbf{T}^{k_{1}}=\mathbf{R}^{k_{1}} / \mathbf{Z}^{k_{1}}$ is constructed from a

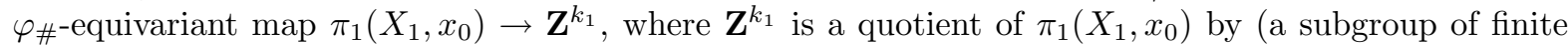
index of) the commutator subgroup (cf. Proposition 3.5, [6]; [20].) The dynamics of the Anosov action are used to $\varphi(\mathcal{A})$-equivariantly extend the quotient map on fundamental groups to a map, from the universal cover of $X_{1}$ to the simply connected nilpotent Lie group $\mathcal{N}$. The abelian Lie group $\mathbf{R}^{k_{1}}$ is identified with the maximal abelian quotient of $\mathcal{N}$, and the induced map is $\pi$. 
Let $x_{1} \in \mathbf{T}^{k_{1}}$ be the image of $x_{0}$, which is a fixed-point for the action of $\varphi_{*}(\mathcal{A})$. (In fact, we can choose the map $\pi$ so that $x_{1}=\overline{0}$ is the coset of the zero vector.) Then the fiber $X_{2}=\pi^{-1}\left(x_{1}\right)$ is a compact nilmanifold, and the induced action of $\varphi(\mathcal{A})$ on $X_{2}$ is hyperbolic with fixed-point $x_{0}$. We can repeat the application of Franks' result to obtain a chain of fibrations

$$
X_{i+1} \subset X_{i} \stackrel{\pi}{\rightarrow} \mathbf{T}^{k_{i}}
$$

which are invariant under the action of $\varphi(\mathcal{A}), x_{0} \in X_{i}$ and with the induced action on $\mathbf{T}^{k_{i}}$ hyperbolic. The total spaces of the fibrations (4) form a chain of compact invariant submanifolds

$$
\left\{x_{0}\right\} \in X_{m} \subset X_{m-1} \subset \cdots \subset X_{1}=X
$$

LEMMA 3.2 For each $1 \leq \ell \leq m$ the induced action $\varphi_{\ell}: \mathcal{A} \times \mathbf{T}^{k_{\ell}} \rightarrow \mathbf{T}^{k_{\ell}}$ on the quotient space $\mathbf{T}^{k_{\ell}} \cong X_{\ell} / X_{\ell+1}$ is Cartan (for an appropriate free abelian subgroup $\mathcal{A}_{\ell} \subset \mathcal{A}$.)

Proof. Parallel to the construction of the sequence of fibrations (4), there is a sequence of subbundles

$$
\mathbf{F}_{m} \subset \mathbf{F}_{m-1} \subset \cdots \subset \mathbf{F}_{1}=T X
$$

which are invariant under the action of $D \varphi(\mathcal{A})$, with $T X_{i}=\mathbf{F}_{i} \mid X_{i}$.

Given $\gamma_{i} \in \mathcal{A}$ with maximally contracting subbundle $\mathbf{E}_{i}$ there is some least index $\nu(i)$ so that $\mathbf{E}_{i} \subset \mathbf{F}_{\nu(i)}$. Then there is a $D \varphi(\mathcal{A})$-invariant internal direct sum decomposition

$$
\mathbf{F}_{\nu(i)}=\mathbf{E}_{i}+\mathbf{G}_{\nu(i)}
$$

where $\mathbf{G}_{\nu(i)}$ is a sum of invariant subbundles $\mathbf{E}_{k}$ for appropriate $k \neq i$. In particular, the action of $D \varphi\left(\gamma_{i}\right)$ on $\mathbf{F}_{\nu(i)}$ admits an exponential dichotomy about $\mu_{i, i}$ with maximally contracting subbundle $\mathbf{E}_{i}$. The invariant subbundle $\mathbf{F}_{\nu(i)+1} \subset \mathbf{F}_{\nu(i)}$ is contained in the complementary bundle $\mathbf{G}_{\nu(i)}$. Therefore, the induced action of $D \varphi\left(\gamma_{i}\right)$ on $T\left(X_{i} / X_{i+1}\right)$ will also satisfy an exponential dichotomy about $\mu_{i, i}$.

Let $B_{i} \in H_{1}\left(X_{\nu(i)} ; \mathbf{R}\right)$ be the homology class of the asymptotic cycle associated to an integral curve for the restriction of $\mathbf{E}_{i}$ to the fiber $X_{\nu(i)}$. This class is non-zero, for the bundle $\mathbf{E}_{i}$ projects to a 1dimensional subbundle of the quotient space $\mathbf{T}^{k_{\nu(i)}}$, whose asymptotic cycle is non-zero in homology. The first homology of the nilmanifold $X_{\nu(i)}$ is the maximal abelian quotient of its fundamental group, hence there is an isomorphism $H_{1}\left(X_{\nu(i)} ; \mathbf{R}\right) \cong H_{1}\left(\mathbf{T}^{k_{\nu(i)}} ; \mathbf{R}\right)$. Thus $B_{i}$ is non-zero, for it is the lift of a non-zero asymptotic class under this isomorphism. By the same method as used to obtain the estimate (3), the asymptotic class $B_{i}$ is an eigenvector for $\varphi\left(\gamma_{i}\right)_{*}$ on $H_{1}\left(X_{\nu(i)} ; \mathbf{R}\right)$ with eigenvalue $\lambda_{i}^{*}$ in the band $\lambda_{i, i} \leq \lambda_{i}^{*} \leq \mu_{i, i}$. It follows that the projection of $B_{i}$ to $\mathbf{T}^{k_{\nu(i)}}$ is an eigenvector for the induced action $\varphi_{\nu(i)}(\mathcal{A})_{*}$ with eigenvalue $\lambda_{i}^{*}$ for the action of $\varphi_{\nu(i)}\left(\gamma_{i}\right)_{*}$.

Now let $\mathcal{A}_{\ell}$ be the abelian group generated by the $\gamma_{i}$ so that $\nu(i)=\ell$. The above established that the induced action $\varphi_{\ell}\left(\mathcal{A}_{\ell}\right)_{*}$ is diagonalized on $H_{1}\left(\mathbf{T}^{k_{\ell}} ; \mathbf{R}\right)$ with a unique maximally contracting eigenspace associated to each $\gamma_{i} \in \mathbf{A}_{\ell}$ as was to be shown.

Proposition 3.1 follows from Lemma 3.2 and the next observation:

LEMMA 3.3 The affine action $\varphi_{\#}: \mathcal{A} \times X \rightarrow X$ is Cartan if the induced action on

$$
\bigoplus_{i=1}^{m} H_{1}\left(X_{i} / X_{i+1} ; \mathbf{R}\right)
$$

is Cartan.

Proof. The Lie algebra $\mathbf{n}$ of the nilpotent Lie group $\mathcal{N}$ covering $X$ admits a filtration

$$
\{0\} \subset \mathbf{n}_{m} \subset \cdots \subset \mathbf{n}_{2} \subset \mathbf{n}_{1}=\mathbf{n}
$$


where $\mathbf{n}_{i+1}$ is the commutator subalgebra of $\mathbf{n}_{i}$. The commutative quotient algebra $\mathbf{a}_{i}=\mathbf{n}_{i} / \mathbf{n}_{i+1}$ is identified with the tangent space of the $\mathbf{T}^{k_{i}}$ and hence to the homology group $H_{1}\left(\mathbf{T}^{k_{i}} ; \mathbf{R}\right) \cong H_{1}\left(X_{i} / X_{i+1} ; \mathbf{R}\right)$. Thus, if the induced action of $\varphi_{\#}(\mathcal{A})$ on $(7)$ has a diagonalization by eigenspaces corresponding to 1 dimensional spaces of maximal contraction for some element of $\mathcal{A}$, then the same holds for the quotient space

$$
\mathbf{a}_{m} \oplus \cdots \oplus \mathbf{a}_{1} \cong \mathbf{n}_{m} \oplus \mathbf{n}_{m-1} / \mathbf{n}_{m} \oplus \cdots \oplus \mathbf{n}_{1} / \mathbf{n}_{2}
$$

which implies that the same holds for the original algebra $\mathbf{n}$.

COROLLARY 3.4 Let $\varphi: \mathcal{A} \times X \rightarrow X$ be a $C^{1}$-Cartan action on an infra-nilmanifold $X$. Then for each $1 \leq i \leq n$, there is a finite collection $\left\{\gamma_{i j}\right\} \subset \mathcal{A}$ so that each diffeomorphism $\varphi\left(\gamma_{i j}\right)$ has a well-defined center-stable foliation $\mathcal{F}_{i j}^{c s}$, and each leaf $L$ of $\mathcal{F}_{i}$ is the intersection of the leaves of the center-stable foliations $\left\{\mathcal{F}_{i j}^{c s}\right\}$ which contain $L$.

Proof. It suffices to show the result for a finite covering of $X$, so we can reduce to the case where $X$ is a nilmanifold, and $\varphi$ is conjugate to a standard affine action $\varphi_{\#}: \mathcal{A} \times X \rightarrow X$ which is Cartan by Proposition 3.1. It then suffices to prove the claim for the affine action $\varphi_{\#}$. Fix $1 \leq i \leq n$, and let $j \neq i$ be given. Then we must show that there exists $\gamma_{i j} \in \mathcal{A}$ so that $\varphi_{\#}\left(\gamma_{i j}\right)$ has exponent $\leq 1$ on $\mathcal{F}_{i}$ and $\varphi_{\#}\left(\gamma_{i j}\right)$ is expanding on $\mathcal{F}_{j}$. It is an easy exercise to check that there exists $p>0$ and $q<0$ so that $\gamma_{i j}=\gamma_{i}^{p} \gamma_{j}^{q}$ has this property.

\section{Coarse expansion rates of a diffeomorphism}

We discuss in this section some basic notions of "coarse geometry" (cf. Gromov [8, 7]), and then define the "expansion rate of a homeomorphism on a topological curve".

Let $V$ and $W$ be complete metric spaces with distance functions $d_{V}$ and $d_{W}$ respectively. A set map $h: V \rightarrow W$ is a coarse isometry if there exists constants $c_{1}, c_{2}, c_{3}>0$ so that for all $x, y \in V$ with $d_{V}(x, y) \geq c_{1}$ we have

$$
c_{2} \cdot d_{V}(x, y) \leq d_{W}(h(x), h(y)) \leq c_{3} \cdot d_{V}(x, y)
$$

This concept has proven to be extremely valuable for the study of the geometry of complete open manifolds, especially those that arise in as coverings of a compact manifold, or as the leaf of a foliation of a compact manifold. The equivalence relation on complete metric spaces generated by coarse isometry is called coarse equivalence.

A subset $N \subset V$ is called a net if there exists constants $C_{1}, C_{2}>0$ so that

- for any pair $x \neq y \in N, d_{V}(x, y) \geq C_{1}$, and

- for any $x \in V$ there exists $y_{x} \in V$ with $d_{V}\left(x, y_{x}\right)<C_{2}$

Nets arise in two contexts in dynamics: as the intersection of a leaf of a foliation with a cross-section (cf. section 2, [14]) and from coverings of compact manifolds. Suppose that $V \rightarrow X$ is a normal covering of a compact Riemannian manifold $X$, with covering group $\Gamma$. Let $d_{V}$ be the length metric determined by the lift of the Riemannian metric to $V$. Choose a compact fundamental domain $K \subset V$ for the left action of $\Gamma$. Choose a point $v_{0} \in K$ in the interior of $K$ and set $N=\Gamma \cdot v_{0}$. Then $N$ is a net in $V$, with $C_{1}$ equal to twice the distance from $v_{0}$ to the boundary of $K$, and $C_{2}$ equal to one-half the diameter of $K$.

A choice of a generating set $\left\{\gamma_{1}, \ldots, \gamma_{n}\right\}$ for a finitely-generated group $\Gamma$ determines a word metric on $\Gamma$. The coarse equivalence class of the metric on $\Gamma$ is independent of the choice of the generating set. We recall two other basic results:

PROPOSITION 4.1 $V \rightarrow X$ is a normal covering of a compact Riemannian manifold $X$, with covering group $\Gamma$ and base-point $v_{0} \in V$. Then the induced map $\Gamma \cong \Gamma \cdot v_{0} \subset V$ is a coarse isometry between $\Gamma$ with the word metric and $V$ with the Riemannian length metric. 
COROLLARY 4.2 Let $H: X \rightarrow X$ be a continuous map which is homotopic to the identity map. For a normal covering $V \rightarrow X$, lift $H$ to a covering map $h: V \rightarrow V$. Then $h$ is a coarse equivalence, for any Riemannian length metric on $V$ determined by a choice of Riemannian metric on $X$.

Proof. The map $H$ induces the identity map on the covering group $\Gamma$, so induces an isometry on the net in $V$ determined by $\Gamma$, and hence a coarse isometry on the ambient space $V$.

The proof of Theorem 1.1 in the non-toral case requires an extension of Corollary 4.2 to the case of leaves of foliations. We recall the relevant results from [11] where the ideas are developed in more detail. A $C^{0,2}$-foliation $\mathcal{F}$ of a paracompact manifold $X$ is a continuous partition of $X$ into tamely embedded $C^{2}$-submanifolds (the leaves) of constant dimension $p$ and codimension $q$. We require that these leaves be locally given as the level sets (plaques) of local coordinate charts. We fix the local defining data:

1. a locally finite covering $\left\{U_{\alpha} \mid \alpha \in \mathcal{A}\right\}$ of $X$.

2. local coordinate charts $\phi_{\alpha}: U_{\alpha} \rightarrow(-1,1)^{n}$, so that each map $\phi_{\alpha}$ admits an extension to a homeomorphism $\tilde{\phi}_{\alpha}: \tilde{U}_{\alpha} \rightarrow(-2,2)^{n}$ where $\tilde{U}_{\alpha}$ contains the closure of the open set $U_{\alpha}$.

3. for each $z \in(-2,2)^{q}$, the preimage $\tilde{\phi}_{\alpha}^{-1}\left((-2,2)^{p} \times\{z\}\right) \subset \tilde{U}_{\alpha}$ is the connected component containing $\tilde{\phi}_{\alpha}^{-1}(\{0\} \times\{z\})$ of the intersection of the leaf of $\mathcal{F}$ through $\phi_{\alpha}^{-1}(\{0\} \times\{z\})$ with the set $\tilde{U}_{\alpha}$.

4. for $z \in \tilde{U}_{\alpha} \cap \tilde{U}_{\beta}$ and $(x, y)=\tilde{\phi}_{\alpha}(z)$, the composition $\tilde{\phi}_{\beta} \circ \tilde{\phi}_{\alpha}: U_{z} \times\{y\} \rightarrow(-2,2)^{p}$ is $C^{2}$ in a neighborhood $U_{z} \subset(-2,2)^{p}$ of $x$, uniformly in the transverse parameter $y \in(-2,2)^{q}$.

The extensibility condition (2) is made to guarantee that the topological structure on the leaves remains tame out to the boundary of the chart $\phi_{\alpha}$. The collection $\left\{\left(U_{\alpha}, \phi_{\alpha}\right) \mid \alpha \in \mathcal{A}\right\}$ is called a regular foliation atlas for $\mathcal{F}$.

The regularity condition (4) on $\tilde{\phi}_{\beta} \circ \tilde{\phi}_{\alpha}$ implies that the leaves have a uniform structure as $C^{2}$ manifolds, so there is a $C^{2}$-Riemannian metric on the tangential distribution $T \mathcal{F}$.

Let $L_{x}$ denote the leaf of $\mathcal{F}$ through $x \in X$, and $\tilde{L}_{x}$ the universal cover of $L_{x}$. If we consider $\tilde{L}_{x}$ as the endpoint homotopy classes of paths emanating from $x$, then the constant path at $x$ determines a canonical basepoint $\tilde{x} \in \tilde{L}_{x}$. For any pair of points $y, y^{\prime} \in \tilde{L}_{x}$, define the leafwise distance $d_{x}\left(y, y^{\prime}\right)$ as the infimum of the lengths of $C^{1}$-paths in $\tilde{L}_{x}$ from $y$ to $y^{\prime}$ with respect to the Riemannian metric on $T L_{x}$ lifted to $\tilde{L}_{x}$. There is also a plaque distance function $D_{x}$ on $\tilde{L}_{x}$ defined in terms of a net induced on $\tilde{L}_{x}$ by a complete transversal to $\mathcal{F}$. The leaf distance function $d_{x}$ is coarse equivalent to $D_{x}$ by Lemma 2.3 , [11]. The plaque distance function $D_{x}$ is topologically defined, hence the coarse equivalence class of the leaf distance function is independent of the smooth structure.

The following result is a special case of Lemma 2.4, [11]:

PROPOSITION 4.3 Let $\mathcal{F}_{i}$ be a $C^{0,2}$-foliation of a compact manifold $X_{i}$ for $i=1,2$ and $f: X_{1} \rightarrow X_{2}$ a continuous map which sends the leaves of $\mathcal{F}_{1}$ into the leaves of $\mathcal{F}_{2}$. Then there exist constants $c_{1}, c_{3}>0$ so that for all $x \in X_{1}$ and $y, y^{\prime} \in \tilde{L}_{x}$ with $d_{x}\left(y, y^{\prime}\right) \geq c_{1}$, the induced map on universal coverings $\tilde{f}_{x}: \tilde{L}_{x} \rightarrow \tilde{L}_{f(x)}^{\prime}$ satisfies the estimate

$$
d_{f(x)}\left(\tilde{f}_{x}(y), \tilde{f}_{x}\left(y^{\prime}\right)\right) \leq c_{3} \cdot d_{x}\left(y, y^{\prime}\right)
$$

That is, the lifted maps $\tilde{f}_{x}$ admit a uniform estimate on their coarse norm.

Next introduce the expansion rates associated to a homeomorphism $\phi: X \rightarrow X$. By a curve we mean a topological embedding $\sigma: \mathbf{R} \rightarrow X$; that is, for each $a<b$ the restriction $\sigma \mid[a, b] \rightarrow X$ is a homeomorphism onto its image. Fix a Riemannian metric on $X$ and give the universal covering $V$ of $X$ the corresponding Riemannian length metric. Choose a lifting $\tilde{\sigma}: \mathbf{R} \rightarrow V$, and a lifting $\tilde{\phi}: V \rightarrow V$ of $\phi$. Define the upper expansion rate of $\phi$ at $\sigma$ to be

$$
\ell[\phi](\sigma)=\sup _{a<b}\left\{\limsup _{\ell \rightarrow \infty} \frac{1}{\ell}\left\{\log d_{V}\left(\tilde{\phi}^{\ell}(\tilde{\sigma}(b)), \tilde{\phi}^{\ell}(\tilde{\sigma}(a))\right)\right\}\right\}
$$


The concept of "expansion rate at $\sigma$ " codifies properties of how the map $\phi$ expands the image of $\sigma$ in the ambient length metric on $X$. We introduce a variation of this notion, which is defined for foliated maps and leafwise curves, based on the expansion rate relative to the leafwise length metric induced on the leaves. Suppose that $\mathcal{F}$ is a $C^{0,2}$-foliation of $X$. A choice of a Riemannian metric on $T \mathcal{F}$ induces a length metric $d_{x}$ on the universal cover $\tilde{L}_{x}$ of the leaf through $x \in X$, and the coarse isometry class of $\tilde{L}_{x}$ is independent of the choice of metric on $T \mathcal{F}$. A homeomorphism $\phi: X \rightarrow X$ is preserves $\mathcal{F}$ if $\phi$ maps leaves of $\mathcal{F}$ to leaves of $\mathcal{F}$. A curve $\sigma: \mathbf{R} \rightarrow X$ is leafwise if there exists a leaf $L_{x}$ of $\mathcal{F}$ which contains the image of $\sigma$. Given a leafwise curve, choose a lifting $\tilde{\sigma}: \mathbf{R} \rightarrow \tilde{L}_{x}$. Recall that $\tilde{\phi}_{y}: \tilde{L}_{y} \rightarrow \tilde{L}_{\phi(y)}$ is the unique lift of $\phi$ to the universal covers of the leaves such that $\tilde{\phi}(\tilde{y})=\widetilde{\phi(y)}$. The upper expansion rate with respect to $\mathcal{F}$ of $\phi$ at a leafwise curve $\sigma$ is defined as

$$
\ell[\mathcal{F}, \phi](\sigma)=\sup _{a<b}\left\{\limsup _{\ell \rightarrow \infty} \frac{1}{\ell}\left\{\log d_{x}\left(\widetilde{\phi}_{x}^{\ell}(\tilde{\sigma}(b)), \tilde{\phi}_{x}^{\ell}(\tilde{\sigma}(a))\right)\right\}\right\}
$$

We make some basic observations about the upper expansion rate. The proof of the first is elementary:

LEMMA 4.4 Let $\sigma_{0}$ be curve, and $\phi: X \rightarrow X$ a homeomorphism. Let $\sigma_{1}(t)=\sigma_{0}(f(t))$ where $f: \mathbf{R} \rightarrow \mathbf{R}$ is a homeomorphism which is a coarse isometry for the standard metric on $\mathbf{R}$. Then

$$
\ell[\phi]\left(\sigma_{1}\right)=\ell[\phi]\left(\sigma_{0}\right)
$$

The proof of the next estimate follows directly from Proposition 4.1:

LEMMA 4.5 Given homeomorphisms $\phi_{1}: X_{1} \rightarrow X_{1}, \phi_{2}: X_{2} \rightarrow X_{2}$, let $H: X_{1} \rightarrow X_{2}$ be a continuous map so that $\phi_{2} \circ H=H \circ \phi_{1}$. Given a curve $\sigma$, then $H \circ \sigma$ is also a curve and

$$
\ell\left[\phi_{2}\right](H \circ \sigma) \leq \ell\left[\phi_{1}\right](\sigma)
$$

Similarly, Proposition 4.3 yields the following estimate:

LEMMA 4.6 Given homeomorphisms $\phi_{1}: X_{1} \rightarrow X_{1}, \phi_{2}: X_{2} \rightarrow X_{2}$, let $H: X_{1} \rightarrow X_{2}$ be a continuous map so that $\phi_{2} \circ H=H \circ \phi_{1}$. Suppose that $\mathcal{F}_{i}$ is a foliation of $X_{i}$ which is invariant under the homeomorphism $\phi_{i}$, and $H$ maps the leaves of $\mathcal{F}_{1}$ into the leaves of $\mathcal{F}_{2}$. Given a leafwise curve $\sigma$ for $\mathcal{F}_{1}$, then $H \circ \sigma$ is a leafwise curve for $\mathcal{F}_{2}$ and

$$
\ell\left[\mathcal{F}_{2} \mid \phi_{2}\right](H \circ \sigma) \leq \ell\left[\mathcal{F}_{1} \mid \phi_{1}\right](\sigma)
$$

The upper expansion rate for a $C^{1}$-curve $\sigma$ always has an estimate above in terms of the norm of $D \phi$ on the tangent vectors $\frac{d}{d t} \sigma(t)$ :

LEMMA 4.7 Let $\sigma: \mathbf{R} \rightarrow X$ be a $C^{1}$-curve, and let $C>0$ be a constant so that

$$
\left\|D \phi\left(\frac{d}{d t}\right)\right\| \leq C \cdot\left\|\frac{d}{d t}\right\|
$$

for all $t \in \mathbf{R}$. Then $\ell[\phi](\sigma) \leq C$.

The same estimate holds in the foliated case. 


\section{Cartan actions on tori}

We first observe that it suffices to prove Theorem 1.1 for the case $X$ is a nilmanifold, the action of $\varphi_{1}$ fixes a point $x_{0} \in X$, the trellis foliations $\mathcal{F}_{i}$ are oriented and the action of each $\gamma_{i} \in \Delta$ is orientationpreserving. This follows from a sequence of reductions: Let $x_{1} \in X$ be a periodic point for the action of an Anosov diffeomorphism $\varphi_{1}\left(\gamma_{i}\right)$, which is then periodic for the action of $\varphi(\mathcal{A})$. There is a power $p>0$ so that each $\varphi_{1}\left(\gamma_{k}^{p}\right)$ fixes $x_{1}$ for all $1 \leq k \leq n$. Choose a covering $\tilde{X} \rightarrow X$ so that $\tilde{X}$ is a nilmanifold, and the lift of each trellis foliation $\mathcal{F}_{1, i}$ is orientable. Choose a point $\tilde{x}_{1}$ which covers $x_{1}$, and a point $\tilde{x}_{0}$ which covers $H\left(x_{1}\right)$, then lift $H$ to the unique homeomorphism $\tilde{H}: \tilde{X} \rightarrow \tilde{X}$ with $\tilde{H}\left(\tilde{x}_{1}\right)=\tilde{x}_{0}$. The action of $\varphi_{1}\left(\gamma_{k}\right)$ lifts to a unique diffeomorphism which fixes $\tilde{x}_{1}$, and similarly for $\varphi_{0}\left(\gamma_{k}\right)$ fixing $\tilde{x}_{0}$. If we assume that $p$ is an even integer, then $\varphi_{1}\left(\gamma_{k}\right)$ is orientation preserving on the lift of each foliation $\mathcal{F}_{1, i}$. So we obtain an action of a the subgroup $\mathcal{A}^{p} \subset \mathcal{A}$ of finite-index on the nilmanifold $\tilde{X}$ for which the hypotheses of the theorem hold. If the lifted trellis foliations on $\tilde{X}$ are preserved by $\tilde{H}$, then the trellis foliations on $X$ will be preserved by $H$.

Proposition 3.1 reduces the proof of Theorem 1.1 to the case where the Cartan action $\varphi_{0}$ is affine: Let $H^{\prime}: X \rightarrow X$ be the Franks-Manning conjugacy of $\varphi_{0}$ to the affine action $\varphi_{\#}$, so that the fixed-point $x_{0}$ is mapped to the coset $\overline{0} \in X$ of the identity element in the nilpotent group $\mathcal{N}$. Then both $H^{\prime} \circ H$ and $\left(H^{\prime}\right)^{-1}$ satisfy the conditions of Theorem 1.1, and if we prove the conclusion for $\left(H^{\prime}\right)^{-1}$ and for the composition $H^{\prime} \circ H$, then it clearly holds for $H$.

We assume for the rest of this section that $\varphi_{1}: \mathcal{A} \times \mathbf{T}^{n} \rightarrow \mathbf{T}^{n}$ is a Cartan $C^{1}$-action, where $\mathcal{A}$ is an abelian group generated by the set $\Delta=\left\{\gamma_{1}, \ldots, \gamma_{n}\right\}$, such that $\varphi_{1}$ is conjugate by a homeomorphism $H: \mathbf{T}^{n} \rightarrow \mathbf{T}^{n}$ to a linear Cartan action $\left(\varphi_{0}, \Delta\right)$ on $\mathbf{T}^{n}$. We will show that, given $1 \leq i \leq n, H$ conjugates the foliation $\mathcal{F}_{1, i}$ to the linear foliation $\mathcal{F}_{0, i}$. (Actually, the reader can check that the proof given below requires only that $\varphi_{1}\left(\gamma_{i}\right)$ is Anosov with strongest stable foliation $\mathcal{F}_{1, i}$ and $\varphi_{0}$ is a linear Cartan action.) The more general case for nilmanifolds is considered in the next section.

Let $L$ be a leaf of $\mathcal{F}_{1, i}$ such that $H(L)$ is not contained in a leaf of $\mathcal{F}_{0, i}$. We will arrive at a contradiction from this assumption. Give $\mathbf{T}^{n}$ an affine flat Riemannian metric so that the trellis foliations $\left\{\mathcal{F}_{0,1}, \ldots, \mathcal{F}_{0, n}\right\}$ are pairwise orthogonal. Choose a base-point $x \in L$, and introduce a parametrization $\sigma: \mathbf{R} \rightarrow \mathbf{T}^{n}$ of $L$ with $\sigma(0)=x$ and $\frac{d}{d t} \sigma(t)$ the positively-oriented unit vector field tangent to $L$. Let $\tilde{\sigma}: \mathbf{R} \rightarrow \mathbf{R}^{n}$ be a lift to the universal covering.

Recall that the spectrum of $D \varphi_{1}\left(\gamma_{k}\right)$ on $\mathbf{E}_{i}$ is pinched between $\lambda_{k, i}$ and $\mu_{k, i}$, and that the rate of maximal contraction for $D \varphi_{1}\left(\gamma_{k}\right)$ on $\mathbf{E}_{k}$ is bounded above by $\mu_{k, k}<\lambda_{k, i}$. We deduce by Lemma 4.7:

LEMMA 5.1

$$
\ell\left[\varphi_{1}\left(\gamma_{k}^{-1}\right)\right](\sigma) \leq \lambda_{k, i}^{-1}
$$

Lemma 4.5 implies that the same upper estimate is true for the image curve $H \circ \sigma$.

$$
\text { COROLLARY 5.2 } \quad \ell\left[\varphi_{0}\left(\gamma_{k}^{-1}\right)\right](H \circ \sigma) \leq\left(\lambda_{k, i}\right)^{-1} \text {. }
$$

Let $\left\{\vec{v}_{1}, \ldots, \vec{v}_{n}\right\}$ be positively-oriented unit-vector fields tangent to these foliations. Then introduce coordinates $\left(r_{1}, \ldots, r_{n}\right)$ on $\mathbf{R}^{n}$ determined by the lift of the vector fields, with $(0, \ldots, 0)$ corresponding to a the origin in $\mathbf{R}^{n}$. The leaves of the foliation $\mathcal{F}_{0, k}$ lift to the lines $\left\{\left(r_{1}, \ldots, r_{k}=t, \ldots, r_{n}\right) \mid t \in \mathbf{R}\right\}$, so that a curve $c: \mathbf{R} \rightarrow \mathbf{T}^{n}$ is contained in a leaf of $\mathcal{F}_{0, i}$ if and only if for a lift $\tilde{c}$, the coordinate functions $r_{k}(\tilde{c}(t))$ are constant in $t$ for all $k \neq i$.

Our assumption is that there exists $k \neq i$ so that the coordinate $r_{k}(\tilde{H}(\tilde{\sigma}(t)))$ is not constant. Hence, there exists points $a<b$ so that $r_{k}(\tilde{H}(\tilde{\sigma}(a))) \neq r_{k}(\tilde{H}(\tilde{\sigma}(b)))$. Set

$$
\begin{aligned}
\vec{z} & =\tilde{H}(\tilde{\sigma}(a))=\left(r_{1}, \ldots, r_{n}\right) \in \mathbf{R}^{n} \\
\vec{z}^{\prime} & =\tilde{H}(\tilde{\sigma}(b))=\left(r_{1}^{\prime}, \ldots, r_{n}^{\prime}\right) \in \mathbf{R}^{n}
\end{aligned}
$$


where $r_{k} \neq r_{k}^{\prime}$. The action of $\varphi_{0}$ on $\mathbf{T}^{n}$ has a canonical lifting to an action on $\mathbf{R}^{n}$ which we will denote simply by $\vec{v} \mapsto \gamma \cdot \vec{v}$ for $\gamma \in \mathcal{A}$. Then we calculate

$$
\begin{aligned}
d_{\mathbf{R}^{n}}\left(\gamma_{k}^{-\ell} \cdot \vec{z}, \gamma_{k}^{-\ell} \cdot \vec{z}^{\prime}\right) & =\left|\left(\lambda_{k, 1}^{*}\right)^{-\ell}\left(r_{1}-r_{1}^{\prime}\right)+\cdots+\left(\lambda_{k, k}^{*}\right)^{-\ell}\left(r_{k}-r_{k}^{\prime}\right)+\cdots+\left(\lambda_{k, n}^{*}\right)^{-\ell}\left(r_{n}-r_{n}^{\prime}\right)\right| \\
& \geq\left(\lambda_{k, k}^{*}\right)^{-\ell}\left|r_{k}-r_{k}^{\prime}\right|-\left|\left(\lambda_{k, 1}^{*}\right)^{-\ell}\left(r_{1}-r_{1}^{\prime}\right)+\cdots+\left(\lambda_{k, n}^{*}\right)^{-\ell}\left(r_{n}-r_{n}^{\prime}\right)\right|
\end{aligned}
$$

where the $k^{t h}$ term is omitted in this last expression. The term $\left(\lambda_{k, k}^{*}\right)^{-\ell}$ is dominant as $\ell \rightarrow \infty$, so we deduce that

$$
\ell\left[\varphi_{0}\left(\gamma_{k}^{-1}\right)\right](H \circ \sigma) \geq\left(\lambda_{k, k}^{*}\right)^{-1} .
$$

This contradicts the conclusion of Corollary 5.2, so that $r_{k} \neq r_{k}^{\prime}$ is impossible and the image of $H(L)$ must lie in a leaf of the strongest stable foliation $\mathcal{F}_{0, i}$. This completes the proof of Theorem 1.1 in the case where $X$ is finitely covered by $\mathbf{T}^{n}$.

The above proof that the image of a leaf of the strongest stable foliation lies in a corresponding strongest stable leaf used three geometric properties: the coarse invariance of the expansion rate of an appropriate Cartan element acting on a unit speed parametrization of the leaf; the existence of diffeomorphisms $\left\{\varphi_{0}\left(\gamma_{k}^{-1}\right)\right\}$ which expand the axes of an orthonormal coordinate system at maximal rates; and the estimation of the expansion rate of an image curve $H \circ \sigma$ via the expansion rate of the Anosov diffeomorphism $\varphi_{0}\left(\gamma_{k}^{-1}\right)$ on the leaves of the strongest unstable foliation $\mathcal{F}_{0, k}$. Implicit in this last estimation is that the distance in the ambient metric on $\mathbf{R}^{n}$ between two points on a strong stable leaf is proportional to the path-length of the leaf segment between them. (This is the property that a linear embedding of $\mathbf{R} \subset \mathbf{R}^{n}$ is straight in the sense of Gromov; that is, the embedding induces a coarse isometry between $\mathbf{R}$ with the standard metric and $\mathbf{R}$ with the induced metric.)

The leaves of the strong stable foliations of an affine Cartan action on an infra-nilmanifold are defined by left-invariant vector fields, but these curves need not be straight in the ambient length metric (associated to a left-invariant Riemannian metric on $T \mathcal{N}$.) This forces the introduction of the use of "straightness relative to a foliation". Applying the above three steps to the expansion rate relative to invariant foliations yields the extension of the above results to the case of Cartan actions on nilmanifolds.

For the torus, the "expansion rate at curves" can be formulated in terms of the expansion of the Cartan action on the Ruelle-Sullivan cohomology classes of the leaves of the trellis, which leads to an alternative proof of the invariance of the trellis for toral actions. The extension of the cohomology method to nilmanifolds involves constructing versions of the Ruelle-Sullivan classes in foliated cohomology. The present proof via methods of "coarse geometry" is more conceptual and direct.

\section{Cartan actions on nilmanifolds}

Let $X=\Lambda \backslash \mathcal{N}$ be a compact nilmanifold with an affine Cartan action $\varphi_{\#}: \mathcal{A} \times X \rightarrow X$ which fixes the coset $\overline{0}$ of the identity element in $\mathcal{N}$. In this section we prove that if $H: X \rightarrow X$ is a topological conjugacy between a $C^{1}$-Cartan action $\varphi_{1}: \mathcal{A} \times X \rightarrow X$ and the affine action $\varphi_{\#}$ then $H$ maps the trellis foliations of $\varphi_{1}$ into those of $\varphi_{\#}$, completing the proof of Theorem 1.1. We will first recall some basic topological properties of nilmanifolds ([18]; Chapter $1[2]$; [20]) then show how this structure enables us to extend the method of the previous section.

Introduce the upper central sequence of connected normal subgroups

$$
\{0\} \subset \mathcal{N}_{m} \subset \cdots \subset \mathcal{N}_{1}=\mathcal{N}
$$

where $\mathcal{N}_{\xi}=\left[\mathcal{N}, \mathcal{N}_{\xi-1}\right]$. This has the property that the quotient $\mathcal{N}_{\xi} / \mathcal{N}_{\xi+1}$ is central in $\mathcal{N} / \mathcal{N}_{\xi+1}$ for all $1 \leq \xi \leq m$. There is a corresponding sequence of normal subgroups of $\Lambda$

$$
\{0\} \subset \Lambda_{m} \subset \cdots \subset \Lambda_{1}=\Lambda
$$


where $\Lambda_{\xi} \subset \mathcal{N}_{\xi}$, the quotient $\Lambda / \Lambda_{\xi}$ is torsion-free, and acts properly discontinuously on the nilmanifold $\mathcal{N} / \mathcal{N}_{\xi}$, with compact quotient manifold denoted by

$$
Q_{\xi}=\left(\Lambda / \Lambda_{\xi+1}\right) \backslash\left(\mathcal{N} / \mathcal{N}_{\xi+1}\right)
$$

Each quotient $\mathcal{N}_{\xi} / \mathcal{N}_{\xi+1}$ is isomorphic to $\mathbf{R}^{c_{\xi}}$ as a Lie group, and the quotient

$$
\left(\Lambda_{\xi} / \Lambda_{\xi+1}\right) \backslash\left(\mathcal{N}_{\xi} / \mathcal{N}_{\xi+1}\right) \cong \mathbf{T}^{c_{\xi}}
$$

is a Lie group isomorphic to a torus. Its natural action on $Q_{\xi}$ defines a fibration of compact nilmanifolds

$$
\mathbf{T}^{c_{\xi}} \rightarrow Q_{\xi} \rightarrow Q_{\xi-1}
$$

Note that $Q_{1} \cong \mathbf{T}^{c_{1}}$ and $Q_{m}=X$.

The above structures are natural with respect to an automorphism of $X$ induced from a Lie group automorphism of $\mathcal{N}$ which preserves the lattice $\Lambda$. In particular, the group action $\varphi_{\#}: \mathcal{A} \times X \rightarrow X$ induces quotient actions

$$
\varphi_{\#}^{\xi}: \mathcal{A} \times Q_{\xi} \rightarrow Q_{\xi}
$$

for all $1 \leq \xi \leq m$ which commute with the factor map $\Pi_{\xi}: X \rightarrow Q_{\xi}$.

We need one more observation about the geometry of the spaces $Q_{\xi}$. Let $D \subset \mathcal{N}$ be a 1-parameter subgroup. Then the image of $D$ in $\mathcal{N} / \mathcal{N}_{\xi+1}$ and $\mathcal{N}_{\xi} / \mathcal{N}_{\xi+1}$ together generate an abelian subgroup of $\mathcal{N}_{\xi} D \subset \mathcal{N} / \mathcal{N}_{\xi+1}$. Give $Q_{\xi}$ the Riemannian metric obtained from the left-invariant Riemannian metric on $\mathcal{N} / \mathcal{N}_{\xi+1}$. Then the leaves of the foliation of $Q_{\xi}$ defined by the right action of $\mathcal{N}_{\xi} D$ on $Q_{\xi}$ - equipped with the restricted Riemannian metric from $Q_{\xi}$ - are isometric to the standard Euclidean plane. In particular, the 1-parameter subgroup $D \subset \mathcal{N}_{\xi} D$ (and any translate of it by an element of $\mathcal{N}_{\xi} D$ ) is a straight curve in the length metric on $\mathcal{N}_{\xi} D$.

Our hypotheses imply that the continuous map $H_{\xi}=H \circ \Pi_{\xi}: X \rightarrow Q_{\xi}$ satisfies

$$
\varphi_{\#}^{\xi}(\gamma) \circ H_{\xi}=H_{\xi} \circ \varphi_{1}(\gamma) \text { for all } \gamma \in \mathcal{A} \text {. }
$$

Now suppose that $L$ is a leaf of $\mathcal{F}_{1, i}$. Give $X$ the Riemannian metric obtained from the left-invariant Riemannian metric on $\mathcal{N}$. Choose a base-point $x \in L$, and introduce a parametrization $\sigma: \mathbf{R} \rightarrow X$ of $L$ with $\sigma(0)=x$ and $\frac{d}{d t} \sigma(t)$ the positively-oriented unit vector field tangent to $L$. Let $\tilde{\sigma}: \mathbf{R} \rightarrow \mathcal{N}$ be a lift to the universal covering.

For each $1 \leq k \leq n$ the spectrum of $D \varphi_{1}\left(\gamma_{k}\right)$ on $\mathbf{E}_{i}$ is pinched between $\lambda_{k, i}$ and $\mu_{k, i}$, and the rate of maximal contraction for $D \varphi_{1}\left(\gamma_{k}\right)$ on $\mathbf{E}_{k}$ is bounded above by $\mu_{k, k}$. Lemma 4.7 implies:

\section{LEMMA 6.1}

$$
\ell\left[\mathcal{F}_{1, i} \mid \varphi_{1}\left(\gamma_{k}^{-1}\right)\right](\sigma) \leq \lambda_{k, i}^{-1} .
$$

The key to the proof in the nilpotent case is to introduce, for each $1 \leq \xi \leq m$ the foliation $\mathcal{N}_{\xi} \mathcal{F}_{0, i}$ of $Q_{\xi}$ with tangential distribution spanned by the tangential distribution to $\mathcal{F}_{0, i}$ and the left-invariant tangent vectors to $\mathcal{N}_{\xi}$. Note that the foliation $\mathcal{F}_{0, i}$ lifted to $\mathcal{N}$ is spanned by a left-invariant vector field on $\mathcal{N}$. Apply Lemma 6.1 and Lemma 4.6 to obtain:

LEMMA 6.2 Suppose that $H_{\xi}$ maps the leaves of $\mathcal{F}_{1, i}$ to the leaves of $\mathcal{N}_{\xi} \mathcal{F}_{0, i}$ for some $1 \leq \xi \leq m$. Then

$$
\ell\left[\mathcal{N}_{\xi} \mathcal{F}_{0, i} \mid \varphi_{0}\left(\gamma_{k}^{-1}\right)\right](H \circ \sigma) \leq\left(\lambda_{k, i}\right)^{-1}
$$

The proof of Theorem 1.1 is by induction. Note that the methods of section 5 apply directly to the map $H_{1}: X \rightarrow Q_{1}=\mathbf{T}^{c_{1}}$ to yield a dichotomy:

- either the image of each leaf of $\mathcal{F}_{1, i}$ is a point in $Q_{1}$, or 
- $\mathcal{F}_{0, i}$ descends to a non-trivial foliation of $Q_{1}$ and the image of each leaf of $\mathcal{F}_{1, i}$ is contained in a leaf of $\mathcal{F}_{0, i}^{1}$.

Next, assume that there is a dichotomy for the map $H_{\xi}: X \rightarrow Q_{\xi}$ :

- either the image of each leaf of $\mathcal{F}_{1, i}$ is a point in $Q_{\xi}$, or

- $\mathcal{F}_{0, i}$ descends to a non-trivial foliation $\mathcal{F}_{0, i}^{\xi}$ of $Q_{\xi}$ and the image of each leaf of $\mathcal{F}_{1, i}$ is contained in a leaf of $\mathcal{F}_{0, i}^{\xi}$.

Then we show that the same dichotomy holds for $H_{\xi+1}$.

The inductive assumption implies that the images of the leaves of $\mathcal{F}_{1, i}$ under the map $H_{\xi+1}$ are contained in the leaves of the foliation $\mathcal{N}_{\xi+1} \mathcal{F}_{0, i}$. Suppose that there is a leaf $L$ of $\mathcal{F}_{1, i}$ whose image under $H_{\xi+1}$ is not a point, and is not contained in a leaf of $\mathcal{F}_{0, i}^{\xi+1}$. We obtain a contradiction from this assumption. The hypotheses of Lemma 6.2 are satisfied by $H_{\xi+1}$, so for each $k \neq i$ there is an estimate

$$
\ell\left[\mathcal{N}_{\xi+1} \mathcal{F}_{0, i} \mid \varphi_{0}\left(\gamma_{k}^{-1}\right)\right](H \circ \sigma) \leq\left(\lambda_{k, i}\right)^{-1}
$$

Let $\mathcal{L}$ denote the leaf of $\mathcal{N}_{\xi+1} \mathcal{F}_{0, i}$ which contains the image of the curve $H_{\xi+1} \circ \sigma$. The tangent space to $\mathcal{L}$ is spanned by commuting left-invariant vector fields on $\mathcal{N} / \mathcal{N}_{\xi+2}$ - the tangents to $\mathcal{F}_{0, i}^{\xi+1}$ and the tangents to $\mathcal{N}_{\xi+1} / \mathcal{N}_{\xi+2}$. Moreover, these vector fields are mapped to multiples of themselves by the induced map $\varphi_{\#}^{\xi+1}(\mathcal{A})$ on $Q_{\xi+1}$. Introduce orthogonal coordinates on $\mathcal{L}$ so that the coordinate axis correspond to the leaves of the foliations $\mathcal{F}_{0, k}^{\xi+1}$ through $H_{\xi+1}(\sigma(0))$. The assumption on $L$ implies that there is some $k \neq i$ so that the $k^{t h}$-coordinate in $\mathcal{L}$ of $H_{\xi+1} \circ \sigma$ is not constant. We can then repeat the calculation of equation (14) to obtain that

$$
\ell\left[\mathcal{N}_{\xi+1} \mathcal{F}_{0, i} \mid \varphi_{0}\left(\gamma_{k}^{-1}\right)\right](H \circ \sigma) \geq\left(\mu_{k, k}\right)^{-1}>\left(\lambda_{k, i}\right)^{-1}
$$

which is a contradiction. This establishes the inductive step.

The proof of Theorem 1.1 is completed by noting first that for $\xi=m, Q_{\xi}=X$ so that $\mathcal{F}_{0, i}^{m}=\mathcal{F}_{0, i}$. The dichotomy asserts in this case that the image of each leaf of $\mathcal{F}_{1, i}$ is contained in a leaf of $\mathcal{F}_{0, i}$. The general infra-nilmanifold case is reduced to the special case where $\varphi_{0}$ is algebraic exactly as in the introduction to section 5 .

\section{$7 \quad$ Some examples}

The first example of an Anosov diffeomorphism on a non-toroidal nilmanifold was given by Smale in his celebrated paper [27], and the construction was extended by Auslander and Scheuneman [3]. Dani [4] gave a general construction of Anosov diffeomorphisms on nil-manifolds, based on arithmetic actions on free k-step nilpotent groups. The construction of Cartan actions is more delicate, for this requires that there exist a sufficiently large collection of commuting Anosov elements. Section 7 of [12] gives an extensive list of examples of Cartan actions on tori, while the thesis of N. Qian [23] gives a similar extensive list of examples of Cartan actions on nilmanifolds. The first example is from his list:

EXAMPLE 7.1 (Theorem 6.1.7 [23]) Let $\Gamma \subset S L(n, \mathbf{Z})$ be a subgroup of finite-index. Then for $n=3$, or for $n \geq 5$, the induced action of $\Gamma$ on a compact quotient $X$ of the free two-step nilpotent Lie group $N(n, 2)$ is Cartan. That is, there is a free abelian subgroup $\mathcal{A} \subset \Gamma$ so that the restriction of the standard action on $X$ to $\mathcal{A}$ is Cartan. 
The second example is elementary, but illustrates an observation of Qian:

EXAMPLE 7.2 Let $A_{1}, \ldots, A_{d} \in S L(2, \mathbf{Z})$ be a collection of hyperbolic matrices. Then the joint action of $\mathbf{Z}^{2 d}$ on $\mathbf{T}^{2 d} \cong \mathbf{T}^{2} \times \cdots \times \mathbf{T}^{2}$ defined by

$$
\left(n_{1}, n_{2}, \ldots, n_{2 d-1}, n_{2 d}\right) \cdot\left(\vec{v}_{1}, \ldots, \vec{v}_{d}\right)=\left(A_{1}^{\left(n_{1}-n_{2}\right)} \cdot \vec{v}_{1}, \ldots, A_{d}^{\left(n_{2 d-1}-n_{2 d}\right)} \cdot \vec{v}_{d}\right)
$$

is Cartan.

The point of this example is that there are many elements in $\mathbf{Z}^{2 d}$ whose action on $\mathbf{T}^{2 d}$ are not Anosov, as they have neutral directions. Yet the action as a whole is Cartan, which can be seen by considering the eigen-spectrum of elements $\left\{A_{1}^{\ell_{1}}, \ldots, A_{d}^{\ell_{d}} \mid \ell_{1} \neq 0, \ldots, \ell_{d} \neq 0\right\}$. Qian's observation is that this phenomenon can also happen for Cartan subactions of irreducible lattices. The simplest example is for an arithmetic lattice $\Gamma \subset O(2,2)_{\mathbf{z}}$ in the non-compact form $O(2,2) \subset$ $S L(4, \mathbf{R})$ whose maximal Cartan subgroup $\mathcal{A} \subset \Gamma$ has real-rank two. The action of the Cartan subgroup $\mathcal{A}$ on $\mathbf{T}^{4}$ has the same dynamical properties as the product action above for $d=2$.

The third example is due to Raphael de la Llavé:

EXAMPLE 7.3 (Section 6 [16]) Let $A \in S L(2, \mathbf{Z})$ and $B \in S L(n, \mathbf{Z})$ be hyperbolic matrices. Let $A$ have eigenvalues $\mu, \mu^{-1}$. Assume that $B$ is a regular semi-simple element; that is, all of its eigenvalues are positive and distinct, and have modulus not equal to 1 . Choose an expanding eigenvector $0 \neq \vec{e}_{u} \in \mathbf{R}^{n}$ for $B$ corresponding to an eigenvalue $\lambda$ pinched by the estimate $\mu>\lambda>1$. Form the 1-parameter family of actions of $\mathbf{Z}$ on $\mathbf{T}^{n+2} \cong \mathbf{T}^{2} \times \mathbf{T}^{n}$, with generator

$$
\phi_{t}((x, y), \vec{v})=\left(A(x, y), B \vec{v}+t \cdot \cos (x) \vec{e}_{u}\right)
$$

- Then $\phi_{0}$ preserves a linear trellis on $\mathbf{T}^{n+2}$

- the Anosov diffeomorphism $\phi_{t}$ preserves a real analytic trellis on $\mathbf{T}^{n+2}$

- for all t sufficiently small, $\phi_{t}$ is Anosov and topologically conjugate to $\phi_{0}$,

- the trellis foliations of $\phi_{t}$ are not conjugated to the trellis foliations of $\phi_{0}$.

The above examples are now part of folklore, due to their simplicity and many remarkable phenomenon they exhibit: It is clear that the perturbation given by (16) preserves the linear exponents of the action at periodic points, as the perturbation consists of "sliding the along an unstable foliation of $B$ " with dependence on the coordinates in the first factor. So these actions give 1-parameter families of Anosov diffeomorphisms with constant exponents.

There is a unique topological conjugacy $h_{t}: \mathbf{T}^{n+2} \rightarrow \mathbf{T}^{n+2}$ between $\phi_{t}$ and $\phi_{0}$ which is isotopic to the identity. De la Llavé shows that $h_{t}$ is of the form

$$
h_{t}((x, y), \vec{v})=\left((x, y), \vec{v}+\psi_{t}(x) \cdot \vec{e}_{u}\right)
$$

for appropriate choice of $\psi_{t}$, and then explicitly calculates the regularity of $\psi_{t}$, showing that it is $C^{r}$ for $r<\log \mu / \log \lambda$. In particular, $h_{t}$ is never real analytic for $t \neq 0$.

If the trellis foliations for $\phi_{t}$ are preserved by a topological conjugacy $h_{t}$, then Theorem 5.1 of [12] implies that the conjugacy is analytic, based on the regularity theorems of $[17,15,16]$. Thus, $h_{t}$ does not map the trellis associated to $\phi_{t}$ for $t \neq 0$ to the linear trellis for $\phi_{0}$.

The strongest stable and unstable foliations of the Anosov diffeomorphism in Example 7.3 are tangent to the factor $\mathbf{T}^{2}$ in the decomposition $\mathbf{T}^{n+2} \cong \mathbf{T}^{2} \times \mathbf{T}^{n}$, and this first factor is explicitly seen to be preserved by the conjugacy $h_{t}$. Thus, the strongest stable foliations are also preserved by $h_{t}$. The following problem remains open:

PROBLEM 7.4 Show that a topological conjugacy $H$ between Anosov $C^{1}$-diffeomorphisms $\varphi_{0}$ and $\varphi_{1}$ on an infra-nilmanifold $X$ with 1-dimensional strongest stable foliations, preserves these foliations. Does the same hold for the flag of the foliations defined by the Lyapunov band decomposition of $\varphi_{0}$ and $\varphi_{1}$ ? 


\section{References}

[1] D. V. Anosov. Geodesic Flows on Closed Riemannian manifolds with negative curvature, volume 90 (1967) of Proc. Steklov Inst. Math. Amer. Math. Soc., 1969.

[2] L. Auslander, L. Green, and F. Hahn. Flows on Homogeneous Spaces. Princeton University Press, Princeton, NJ, 1963.

[3] L. Auslander and J. Scheuneman. On certain automorphisms of nilpotent Lie groups. In Global Analysis, pages 9-15, Providence, R. I., 1971. Amer. Math. Soc. Proc. Symp. Pure Math. 14.

[4] S. G. Dani. Nilmanifolds with Anosov automorphism. Jour. London Math. Soc., 18:553-559, 1978.

[5] J. Franks. Anosov diffeomorphisms on tori. Trans. Amer. Math. Soc., 145:117-124, 1969.

[6] J. Franks. Anosov diffeomorphisms. In Global Analysis, pages 9-15, Providence, R. I., 1971. Amer. Math. Soc. Proc. Symp. Pure Math. vol 14.

[7] M. Gromov. Hyperbolic manifolds, groups and actions. In I. Kra and B. Maskit, editors, Riemann surfaces and related topics, Stony Brook Conference 1978, Princeton, NJ, 1981. Princeton Univ. Press. Annals of Math. Studies vol. 97.

[8] M. Gromov. Asymptotic invariants of infinite groups. 1992. IHES Preprint M/92/8.

[9] S. G. Hancock. Orbits of paths under hyperbolic toral automorphisms. In Dynamical Systems I - Warsaw, pages 93-96, Paris, 1977. Société Mathématique de France. Astérisque No. 49.

[10] M. W. Hirsch. On invariant subsets of hyperbolic sets. In A. Haefliger and R. Narasimhan, editors, Essays on Topology and Related Topics, pages 126-135. Springer-Verlag, 1970.

[11] S. Hurder. Exotic index theory for foliations. preprint, 1992.

[12] S. Hurder. Rigidity for Anosov actions of higher rank lattices. Annals of Math., 135:361-410, 1992.

[13] S. Hurder. Affine Anosov actions. Mich. Math. Jour., 40, 1993.

[14] S. Hurder and A. Katok. Ergodic theory and Weil measures for foliations. Annals of Math., 126:221-275, 1987.

[15] S. Hurder and A. Katok. Differentiability, rigidity and Godbillon-Vey classes for Anosov flows. Publ. Math. Inst. Hautes Etudes Sci., 72:5-64, 1990.

[16] R. de la Llavé. Smooth conjugacies and SRB measures for uniformly and non-uniformly hyperbolic systems. Comm. Math. Phys., 1992. IHES Preprint M/91/30.

[17] R. de la Llavé, J. Marco, and R. Moriyon. Canonical perturbation theory of Anosov systems and regularity results for Livsic cohomology equation. Annals of Math., 123:537-612, 1986.

[18] A. I. Mal'cev. On a class of homogeneous spaces. Izv. Akad. Nauk. SSSR Ser. Mat., 13:9-32, 1949. English transl., Amer. Math. Soc. Transl. (1) 9 (1962), 276-307.

[19] A. Manning. There are no new Anosov diffeomorphisms on tori. American Jour. Math., 96:422-429, 1974.

[20] A. Manning. Toral automorphisms, topological entropy and the fundamental group. In Dynamical Systems II - Warsaw, pages 273-281, Paris, 1977. Société Mathématique de France. Astérisque No. 50.

[21] S. Newhouse. On codimension one Anosov diffeomorphisms. American Jour. Math., 92:761-770, 1970.

[22] J. Plante. Foliations with measure-preserving holonomy. Annals of Math., 102:327-361, 1975.

[23] N. Qian. Rigidity Phenomenon of Group Actions on a Class of Nilmanifolds and Anosov $\mathbf{R}^{n}$ Actions. PhD thesis, California Institute of Technology, 1992.

[24] D. Ruelle and D. Sullivan. Currents, flows and diffeomorphisms. Topology, 14:319-327, 1975.

[25] S. Schwartzman. Asymptotic cycles. Annals of Math., 66:270-284, 1957.

[26] M. Shub. Global Stability of Dynamical Systems. Springer-Verlag, New York and Berlin, 1987.

[27] S. Smale. Differentiable dynamical systems. Bulletin Amer. Math. Soc., 73:747-817, 1967. 